\title{
A numerical investigation on a capsule-intake of the electrical submersible pump in skid
}

André Damiani Rocha ${ }^{1, *}$, Guilherme Alonso Solano ${ }^{1}$, Daniel Jonas Dezan ${ }^{1}$, William Monte Verde ${ }^{2}$, Jorge Luiz Biazussi ${ }^{2}$, and Antonio Carlos Bannwart ${ }^{3}$

\footnotetext{
${ }^{1}$ Laboratory of Simulation and Modeling on Energy Engineering, Federal University of ABC, Santo André, 09210-580 SP, Brazil

${ }^{2}$ Center for Petroleum Studies, University of Campinas, Campinas, 13083-970 SP, Brazil

${ }^{3}$ School of Mechanical Engineering of Campinas, University of Campinas, Campinas, 13083-970 SP, Brazil
}

Received: 29 October 2020 / Accepted: 9 February 2021

\begin{abstract}
The Electrical Submersible Pumping (ESP) system is one of the most commonly used artificial lift methods in the petroleum industry and one recent breakthrough in this system is enclosed in a capsule and positioned on the seabed in a skid. As it is a recent technology, with only a small amount of equipment currently in operation, there is a clear need for a greater understanding of the flow within this geometry with the objective of perfecting the design of this promising submarine boosting system. This paper presents a numerical investigation of single-phase flow within the scaled capsule-intake of an ESP system in the Skid considering a model with geometric and dynamic similarities in relation to a real equipment in operation in the Espadarte field, located in the Campos Basin, Brazil. The tridimensional and transient simulation for a case for one mass flow rate and inclination angles are investigated. While neglecting the effects of the diffuser and impeller in the system, the flow field features, axial and radial velocity profiles in the intake region were computed. The numerical results show that the flow in the intake region is dominated by the secondary flow, leading to the formation of bathtub vortex. It is expected that the vortices influence the flow pattern in the intake region, breaking the larger bubbles into smaller bubbles, making the transition from the slug flow pattern to the dispersed bubbles or bubbly pattern in which it would be more difficult to be dragged into the intake.
\end{abstract}

\section{Nomenclature}

$d$

$d_{h}$

$I_{\mathrm{T}}$

$k$

$L$

$\bar{p}$

$Q$

$q_{\text {in }}$

$r$

$r / S$

Re

$S$

$S_{i j}$

$U_{b}$

$u_{i}$

$W_{\text {in }}$

$y^{+}$
Tube diameter, $\mathrm{m}$

Hydraulic diameter, $m$

Turbulent intensity

Turbulence kinetic energy, $\mathrm{m}^{2} / \mathrm{s}^{2}$

Length, $\mathrm{m}$

Mean pressure, $\mathrm{Pa}$

Second invariant of velocity gradient tensor, $1 / \mathrm{s}^{2}$

Inlet flow rate, $\mathrm{m}^{3} / \mathrm{s}$

Radial distance, $\mathrm{m}$

Dimensionless radial position

Reynolds number

Magnitude of strain rate, $1 / \mathrm{s}$

Strain rate tensor

Bulk velocity, $\mathrm{m} / \mathrm{s}$

Mean velocity components $(i=1,2,3), \mathrm{m} / \mathrm{s}$

Inlet mass flow rate, $\mathrm{kg} / \mathrm{s}$

Dimensionless distance from the wall

\section{Greek letters}

$\begin{array}{ll}\lambda_{G} & \text { Gas void fraction } \\ \mu_{t} & \text { Eddy viscosity, Pa s } \\ \sigma_{k} & \text { Turbulent Prandtl number } k \\ \sigma_{k 1}, \sigma_{k 2}, \sigma_{\omega 1}, & \text { Constants of the SST } k-\omega \text { models } \\ \sigma_{\omega 2}, \beta_{1}, \beta_{2}, & \\ \beta^{*}, \alpha_{1} & \\ \sigma_{\omega} & \text { Turbulent Prandtl number } \omega \\ \Omega & \text { Vorticity rate, } \mathrm{s}^{-1} \\ \alpha & \text { Inclination angle, degree } \\ \mu & \text { Molecular dynamic viscosity, Pa s } \\ \rho & \text { Fluid density, } \mathrm{kg} \mathrm{m}^{-3} \\ \tau & \text { Shear stress, } \mathrm{N} \mathrm{m}^{-2} \\ \omega & \text { Specific dissipation rate, } \mathrm{s}^{-1}\end{array}$

\section{Introduction}

Artificial lift methods are techniques used to supplement the energy of the oil contained in the reservoir, and they

* Corresponding author: a.damiani@ufabc.edu.br 
are responsible for increasing or even rendering a viable well production. The ESP system is one of the most used artificial lift methods, mainly characterized by the high potential of production and flexibility and suitable for onshore and offshore fields, in vertical or directional wells. The components of the ESP system (i.e., electric motor, seal, multistage centrifugal pump, power cable and monitoring sensors) are submersed and placed at the well bottom. Each stage of the pump has a rotating and stationary component, which are the impeller and the diffuser, respectively. Zhu et al. (2019) highlight the importance of the ESP system and classify this artificial lifting method as the second most used worldwide and the first one with regard to the production volume.

The main advantages of positioning the ESP inside the well include flow performance and flow assurance aspects, since the pressure and temperature are the highest possible. Thus, the produced fluid will have the lowest viscosity and fraction of free gas. However, this creates a major disadvantage for the method. When the system fails, a specific workover rig is needed to intervene in the well and replace the components of the set. In general, the availability of this rig is limited in the supply chain and is very costly.

In this context, to maintain the advantages of the ESP system and mitigate its downsides, a recent improvement in submarine pumping technology is the Electrical Submersible Pump in Skid (ESP-S). In this system, a conventional ESP system is enclosed in a capsule and positioned on the seabed in a skid. This circumvents the need for a specific rig to perform the workover operation, as it is no longer necessary to remove the entire production column and lower it again. The launch of the ESP-S system can be carried out by cables, using simpler and cheaper vessels in reduced time. In a common design, the ESP-S system has two pumps, one on each capsule, which are connected hydraulically in series and powered in parallel (Tarcha et al., 2015).

The disadvantages of the ESP-S are the lower oil pressure and temperature in the pump intake, when compared to the conventional ESP installed in the well. Thus, the fraction of free gas can increase significantly and, as the capsule does not have a gas drain system, operational instabilities may occur. As it is a recent technology, with few equipment currently in operation, there is a clear need for a greater understanding of the flow within this geometry with the objective of perfecting the design of this promising submarine boosting system.

Studies have shown advantages of the ESP-S when compared to other ESP's layouts (Costa et al., 2013). Tarcha et al. (2015) also concluded that the ESP-S has installation advantages and lower maintenance costs. Silva (2010) developed a two-phase flow study of water and gas in a Pumping Module (MOBO) installation, which is another variation of the ESP, by using Computational Fluid Dynamic (CFD) techniques and the results were compared to experimental results obtained by CENPES/Petrobras. The numerical model represented showed good agreement when compared with the experimental results. Additionally, the author concluded that the geometric aspects of the equipment are contribution factors for the establishment of the phases.

There are many experimental and numerical studies of fluid flow within an ESP diffuser-impeller where, for example, the influence of gas or viscous fluids on system performance, flow patterns, or a behavior of drop motion have been investigated (Monte Verde et al., 2017; Perissinotto et al., 2019a, 2019b; Pineda et al., 2016; Vieira, 2018; Zhu et al., 2016, 2019).

Upstream of the ESP intake, the flow in the capsule is similar to the flow in concentric tubes and several experimental and numerical investigations were performed for turbulent flows (Azouz and Shirazi, 1998; Chung and Sung, 2005; Chung et al., 2002; Neto et al., 2011; Nouri et al., 1993; Okumura et al., 2006; Torii and Yang, 1994; Van Zyl et al., 2013) with focus on obtaining velocity distribution and validating turbulence models.

Close to the intake region, the flow assumes a different behavior than the one downstream. One portion of the fluid passes through the orifices while the other portion remains in the annular tube. In gas-liquid flow, instabilities can occur due to fluid segregation, degrading the pump's performance. Recently, studies of single-phase and two-phase gasliquid flow in the annular region of an ESP-S have been carried out. Vieira (2018) and Vieira et al. (2020) developed an experimental apparatus and used it to investigate the gas-liquid separation in the two-phase intermittent flow in the annular space formed between the pump and the capsule at an inclination angle of $5^{\circ}$, proposing a radial slip model. Using the same experimental apparatus, Custódio (2020) investigated the influence of liquid viscosity and inclination on fluid segregation in a gas-liquid flow. Considering five different inclinations and five viscous solutions, the author estimated the separation efficiency and adjusted the radial slip model proposed by Vieira (2018). Both authors used pressure measurements and image processing as an analysis technique. The initial tests carried out by Custódio (2020) considered single-phase flow with water as the working fluid. The objective of performing single-phase tests was to obtain results for future numerical validation.

Although ESPs are widely employed, studies on the three-dimensional flow field features at the capsule-intake flow were not found in the literature. A single-phase investigation is relevant to increase the understanding of the flow in the intake orifices that precede the first stage of the pump. On that region, the unsteady secondary flow is predominant and some recirculation zones appear, which impose flow instabilities and can influence the operational conditions and natural separation of fluids under a multiphase flow in production operations.

In this paper, CFD techniques were used to simulate the single-phase turbulent unsteady flow in a capsule intake region of an ESP-S. It is a fundamental study, aimed at understanding the physical phenomena involved and characterize the flow pattern. The pressure field, axial and radial velocities profiles were numerically obtained at the intake region using RANS approach with the Shear Stress Transport $k-\omega$ model. The numerical model is validated with the experimental results: annular velocity profiles by Nouri et al. (1993) and pressure drop 


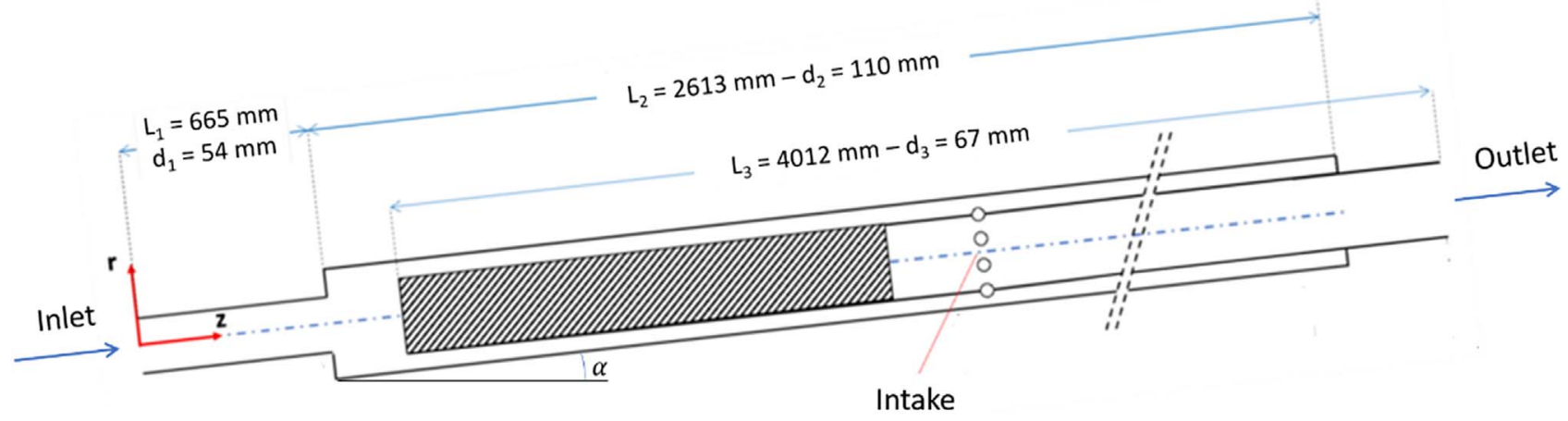

Fig. 1. Computational domain.

measurements by Custódio (2020). Moreover, the effects of secondary flow patterns and vortex motion at the capsuleintake on natural segregation and gas lock are discussed.

\section{ESP-S model}

The computational domain used in this work has the same dimensions as the experimental apparatus developed by Vieira et al. (2020) for the purpose of investigating the gas-liquid separation in the intermittent two-phase flow in the annular region. The experimental apparatus was developed considering geometric and dynamic similarities in relation to a real equipment in operation at Petrobras in the Espadarte field located in the Campos Basin, Brazil. More details on the dimensional analysis can be found in Vieira (2018). Thus, the use of the same geometry ensures that the numerical results predicted in the present study are physically realistic with the real scale of an ESP-S.

The ESP-S flow domain consists of three parts as shown in Figure 1. The first is the inlet pipe of $665 \mathrm{~mm}$ length and $54 \mathrm{~mm}$ i.d., followed by the annular section pipe that starts at $739 \mathrm{~mm}$ from the inlet. The pipe works as a capsule in which its extremity is closed. The annular section pipe has $67 \mathrm{~mm}$ i.d. and $110 \mathrm{~mm}$ i.d. for inner and outer pipe, respectively. The intake, located $1900 \mathrm{~mm}$ from the inlet, has six orifices of $23 \mathrm{~mm}$ i.d., uniformly distributed in the inner tube perimeter with an angle of $60^{\circ}$ among them. The outlet flow is located at $4859 \mathrm{~mm}$ from the inlet section. The ESP-S is housed on the seabed, and it is common for it to be installed with a slight inclination $(\alpha)$ from the horizontal position.

In a real ESP or ESP-S, the electric motor and motor protector are installed in the hatched area, as shown in Figure 1. In this numerical investigation, the hatched area is a solid region and is not part of the computational domain. The shaft and pump were also not considered and the fluid flows to the system through an external motor-pump assembly. The motor will not influence the isothermal flow and the heat dissipation should not influence the analysis. The motor shaft can affect the results in the region of intake, but it was not considered here since the experimental study used for the validation did not consider the motor shaft either. The experimental apparatus was designed to study the separation between gas and liquid that occurs in the annular formed between the pump and the case. With this, it becomes more important to have an experimental apparatus in scale, than the representation of a moving shaft in the intake. Since the intake is located upstream of the pump in a real ESP, the main flow behavior is not affected by the absence of the pump.

The simplification of single-phase flow has some advantages over two-phase flow and the main one is computational cost. The interaction between the gas and liquid phases requires refined computational mesh making processing computationally expensive. In general, the modeling of complex two-phase flows requires knowing the flow pattern. The existing numerical models do not cover, in a single model, the different flow patterns that can be found in ESP-S. Vieira (2018) and Custódio (2020) observed that the intermittent flow pattern was the most commonly found in the annular region and observed the transition from slug flow to dispersed bubbles with increasing liquid flow. Based on this, the single-phase simulation reduces the computational time and can provide critical information for a good understanding of the flow behavior in the annular capsule-intake region.

\section{Numerical approach}

In this paper, the three-dimensional, unsteady, isothermal and incompressible flow is resolved using commercial CFD package Ansys ${ }^{\circledR}$ Fluent 19.0. Numerical simulations of single-phase flow in the capsule-intake region of the ESP-S with Shear Stress Transport (SST) $k-\omega$ turbulence model proposed by Menter (1994), to solve the unsteady three-dimensional incompressible Navier-Stokes equations.

\subsection{Turbulence model}

$$
\begin{gathered}
\frac{\partial \rho}{\partial t}+\frac{\partial}{\partial x_{j}}\left(\rho u_{i}\right)=0 \\
\frac{\partial}{\partial t}\left(\rho u_{i}\right)+\frac{\partial}{\partial x_{j}}\left(\rho u_{i} u_{j}\right)=-\frac{\partial p}{\partial x_{i}}+\frac{\partial}{\partial x_{j}}\left[\mu\left(\frac{\partial u_{i}}{\partial x_{j}}+\frac{\partial u_{j}}{\partial x_{i}}\right)\right] \\
+\frac{\partial}{\partial x_{j}}\left(-\rho \overline{u_{i}^{\prime} u_{j}^{\prime}}\right)
\end{gathered}
$$


For the turbulence $k-\omega$ SST model choice, turbulent studies with similar geometry were considered (Menter, 1994; Neto et al., 2011; Shi et al., 2018; Zhang et al., $2018,2019)$, which prove the precision and quality of the results of the SST model for turbulent flows with a high adverse pressure gradient. In the $k-\omega$ SST turbulence model, turbulent kinetic energy $(k)$ and specific dissipation rate $(\omega)$ are used for close system equations and are defined as follows:

$$
\begin{aligned}
\frac{\partial}{\partial x_{i}}\left(\rho k u_{i}\right)= & \tau_{i j} \frac{\partial u_{i}}{\partial x_{j}}-\rho \beta^{*} k \omega+\frac{\partial}{\partial x_{j}}\left[\left(\mu+\sigma_{k} \mu_{t}\right) \frac{\partial k}{\partial x_{j}}\right] \\
\frac{\partial}{\partial x_{i}}\left(\rho \omega u_{i}\right)= & \frac{\gamma}{v_{t}} \tau_{i j} \frac{\partial u_{i}}{\partial x_{j}}-\rho \beta \omega^{2}+\frac{\partial}{\partial x_{j}}\left[\left(\mu+\sigma_{\omega} \mu_{t}\right) \frac{\partial \omega}{\partial x_{j}}\right] \\
& +2\left(1-F_{1}\right) \frac{\rho \sigma_{\omega 2}}{\omega} \frac{\partial k}{\partial x_{j}} \frac{\partial \omega}{\partial x_{j}}
\end{aligned}
$$

where,

$$
\begin{gathered}
v_{t}=\frac{k}{\omega} \\
\tau_{i j}=\mu_{t}\left(2 S_{i j}-\frac{2}{3} \frac{\partial u_{k}}{\partial x_{k}} \delta_{i j}\right)-\frac{2}{3} \rho k \delta_{i j}, \\
S_{i j}=\frac{1}{2}\left(\frac{\partial u_{i}}{\partial x_{j}}+\frac{\partial u_{j}}{\partial x_{i}}\right), \\
\mu_{t}=\frac{\rho \alpha_{1} k}{\max \left(\alpha_{1} \omega, \Omega F_{2}\right)}, \\
F_{1}=\tan h\left(\arg _{1}^{4}\right), \\
\left.\arg _{1}=\min _{\max }\left(\frac{\sqrt{k}}{\beta^{*} \omega d}, \frac{500 v}{\omega d^{2}}\right), \frac{4 \rho \sigma_{\omega 2} k}{C D_{k \omega} d^{2}}\right] \\
\max _{2}=\tan h\left(2 \rho \sigma_{\omega 2} \frac{1}{\omega} \frac{\partial k}{\partial x_{i}} \frac{\partial \omega}{\partial x_{i}}, 10^{-20}\right), \\
\left.\arg _{2}^{2}\right), \\
\max \left(2 \frac{\sqrt{k}}{\beta^{*} \omega d}, \frac{500 v}{\omega d^{2}}\right) .
\end{gathered}
$$

The constants used for calculation of the parameters described above are: $\sigma_{k 1}=0.85, \sigma_{k 2}=1, \sigma_{\omega 1}=0.65$, $\sigma_{\omega 2}=0.856, \beta_{1}=0.075, \beta_{2}=0.0828, \beta^{*}=0.09$ and $\alpha_{1}=0.31$.

\subsection{Numerical method}

Ansys ${ }^{\circledR}$ Fluent is a software based on finite volume discretization. In this formulation, the computational domain is divided into a given number of volumes, such that the neighborhood for each point in the mesh is composed of control volumes. The governing equations are then integrated into each control volume, obtaining a set of algebraic equations to be solved. For the pressure-velocity coupling the well-known Semi-Implicit Method for Pressure Linked Equations (SIMPLE), the algorithm of Patankar and Spalding (Patankar, 1980, 1981; Patankar and Spalding, 1972) was used. The solution procedure is based on reformulating the Navier-Stokes equations on terms of a momentum and pressure equation, which are then discretized and solved sequentially. The second order upwind discretization scheme has been chosen for pressure, momentum and turbulence equations due to its high-order discretization, which minimizes numerical diffusion errors and offers better accuracy than the first-order discretization schemes. The second order time marching scheme has also been chosen for the integration of the transient term for better accuracy, and a time step of $10^{-4}$ has been used. The computational convergence criteria are ensured when the residuals are lower than $10^{-5}$.

\subsection{Computational domain, grid generation and boundary conditions}

As Figure 1 shows, the ESP-S geometry is a full threedimensional model. In this work, non-uniform hexahedral mesh is generated by using Ansys ${ }^{\circledR}$ Meshing ${ }^{\circledR}$ software. The SST model used modeling close to the wall known as Enhanced Wall Treatment (EWT). For the EWT model, each cell centroid adjacent to the wall must be located within the viscous sublayer and combines a mixed wall law model and a two-layer zonal model. A fine mesh is generated near the walls, keeping the first layer wall elements with $y^{+}$less than unity $\left(y^{+}<1\right)$ and high-quality criteria for skewness and aspect ratio.

As the characterization of the flow at the capsule-intake region is the main objective of the study, the grid has different element sizes through the domain where the intake region is the most refined location. Figure 2 shows a transversal section of the grid at the intake region, indicating the six orifices.

To ensure that the simulation results are mesh independent, the grid independence test was carried out using the Grid Convergence Index (GCI) method (Roache, 1994), in which three meshes and generalized Richardson extrapolation are used to compute and report discretization errors. Three different grids and the total pressure drop were used to verify the independence of the results according to the grid refinements. Table 1 shows the GCI results.

The pressure drop is expressed as $\Delta p=\bar{p}_{\text {out }}-\bar{p}_{\text {in }}$ and average pressure $\bar{p}_{\text {out }}$ and $\bar{p}_{\text {in }}$ was calculated as,

$$
\bar{p}=\frac{\int_{A} p \mathrm{~d} A}{\int_{A} \mathrm{~d} A} .
$$

As observed in Table 1, the relative error for the refined mesh was approximatively $1.4 \%$, which can be considered sufficient for the purposes of this work.

Boundary conditions must be specified in order to solve the governing equations. At the inlet, the mass flow rate is set and it is equal to $33539 \mathrm{~kg} / \mathrm{h}$. This mass flow rate is equivalent to the Reynolds number of 275000 , based on 


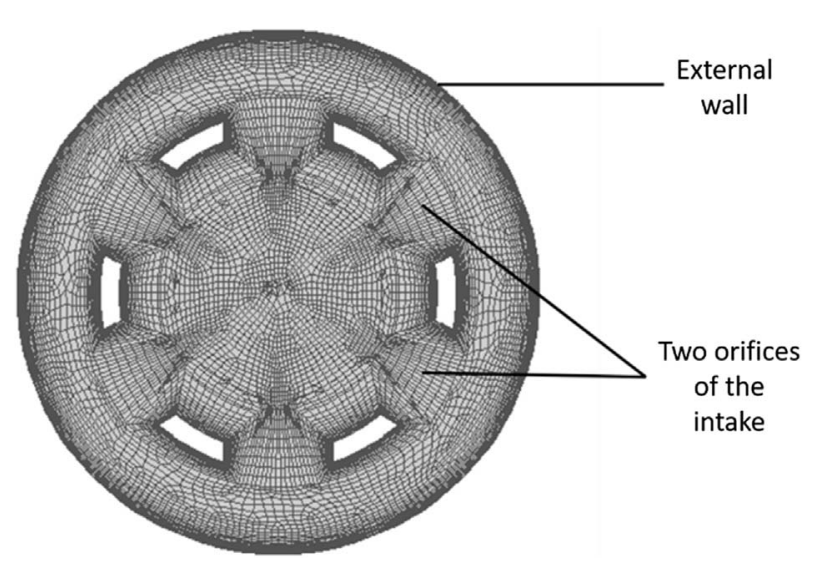

Fig. 2. Grid at the intake region showing the six orifices.

the hydraulic diameter of pipe inlet. At the outlet, the pressure was specified. The no-slip condition was applied at the solid walls. Moreover, the turbulent intensity and the hydraulic diameter were also considered as boundary conditions at the inlet and outlet boundaries of the domain,

$$
I_{T}=0.1 \operatorname{Re}^{-1 / 8} \text {. }
$$

The flow conditions are characterized by the Reynolds number,

$$
\mathrm{Re}=\frac{\rho U_{b} d_{h}}{\mu},
$$

where $U_{b}$ is the mean velocity, $d_{h}$ is the hydraulic diameter (for the inlet it is the inlet tube diameter and, for the outlet, it is the outlet tube diameter) and $\mu$ is the viscosity of the fluid. The numerical run is summarized in Table 2.

\section{Validation}

In order to validate the numerical model used in the current research, numerical pressure drop results were compared with single-phase pressure drop measurements reported by Custódio (2020), who used the same experimental apparatus as Vieira (2018) to investigate the influence of the inclination system and liquid viscosity on the gas-liquid flow in the annular duct. Vieira et al. (2020) presented an experimental campaign to measure the characteristics of intermittent slug flow on the same apparatus used to collect this data, where a great description of this apparatus is presented. It is unnecessary to represent it in this work. A set of single-phase and two-phase results were obtained by the authors and pressure measurements were obtained at four P-station locations named herein as 1-4 (or PT1AD, PT2IN, PT3OP, and PT4OUT), as displayed in Figure 3.

Thus, P-station 1 (PT1AD) is located upstream of the capsule expansion, P-station 2 (PT2IN) is located upstream of the pump intake, P-station 3 (PT3OP) is located at the end of the capsule annular and P-station 4 (PT4OUT) is located at the outlet inner tube. Figure $4 \mathrm{a}$ presents the comparison between the numerical and experimental results. Good agreement between the experimental and
Table 1. Grid independence study.

\begin{tabular}{lccc}
\hline Grid $(-)$ & Cell number $(-)$ & $\Delta p(\mathrm{~Pa})$ & GCI $(\%)$ \\
\hline Coarse & 14940869 & 25981.52 & - \\
Intermediate & 17111290 & 25639.91 & 3.487 \\
Refined & 23669393 & 25205.75 & 1.394 \\
\hline
\end{tabular}

Table 2. Flow conditions and fluid properties.

\begin{tabular}{lc}
\hline Parameter & Quantity \\
\hline Mass flow rate $(\mathrm{kg} / \mathrm{h})$ & 33539 \\
Working fluid & Water \\
Density $\left(\mathrm{kg} / \mathrm{m}^{3}\right)$ & 995.7 \\
Viscosity $(\mathrm{Pa} \mathrm{s})$ & 0.000799 \\
Reynolds number ${ }^{\mathrm{a}}(-)$ & 274825 \\
Reynolds number ${ }^{\mathrm{b}}(-)$ & 80246 \\
Turbulence intensity at the inlet (\%) & 3.344 \\
Turbulence intensity at the outlet $(\%)$ & 3.435 \\
\hline a Based on the hydraulic diameter of inlet pipe. \\
b Based on the hydraulic diameter of annular section.
\end{tabular}

the numerical values of pressure was found, and the maximum relative error is roughly $5 \%$.

Since velocity field measurements were not performed in the single-phase study carried out by Custódio (2020), another validation test was performed using the experimental results of Nouri et al. (1993). The authors experimentally investigated the velocity distribution in a fully developed concentric annulus of a Newtonian fluid at bulk-flow of the Reynolds number 26 000. A periodic boundary condition was imposed in the axial direction by a mass flow rate, and a non-slip boundary condition was imposed at the solid walls. For a computational length of $505 \mathrm{~mm}$, inner tube with $20.0 \mathrm{~mm}$ i.d. and outer tube with $40.3 \mathrm{~mm}$ i.d., measurements of axial, radial and tangential velocity were performed. Figure $4 \mathrm{~b}$ shows the axial velocity distribution weighted by flow average velocity $\left(u / U_{b}\right)$ as a function of the annular gap $(r / S)$, the error bars correspond to $5.5 \%$. The numerical results demonstrate a good agreement with the experimental results. Thus, from the grid independence study and numerical validation, the numerical procedure is assumed to be valid and accurate for the purposes of the present research.

\section{Results and discussion}

\subsection{Flow field analysis}

The numerical velocity and pressure predictions were obtained using the temporal average. A critical region for the flow is the intake region that is constituted by six orifices. In those orifices, part of the fluid flows into the pump region and then goes to the outlet, and the other part remains at the annular section downstream of the orifices. To improve the flow characteristics in the intake region, 


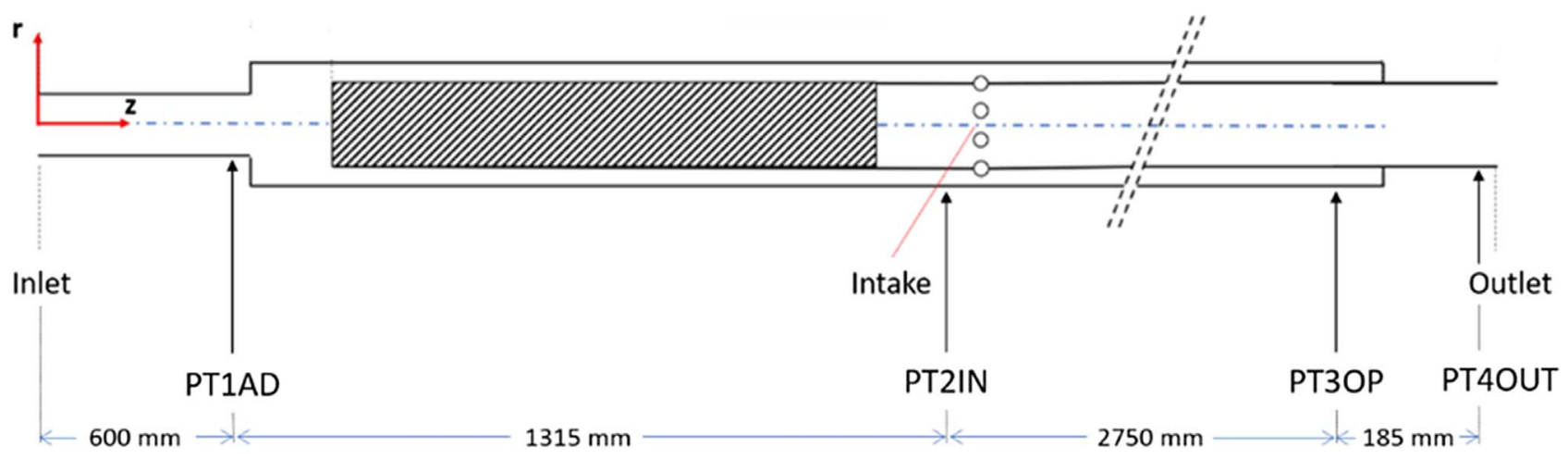

Fig. 3. P-stations used for pressure measurements.

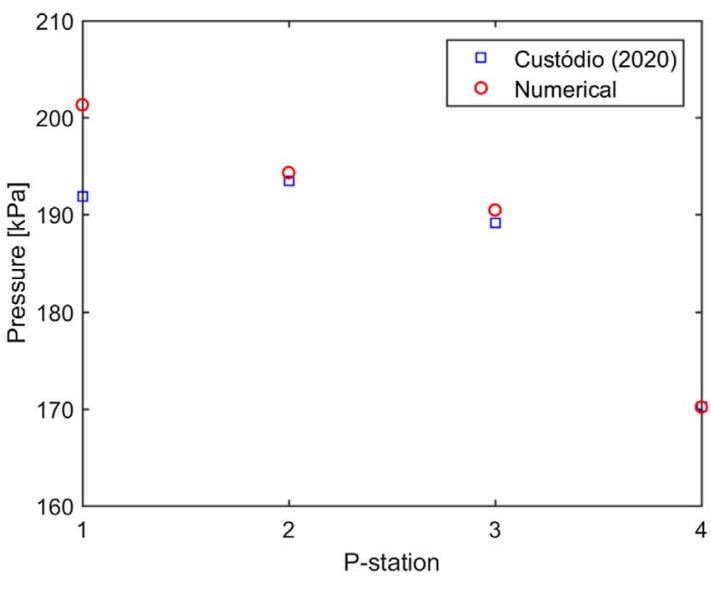

(a)

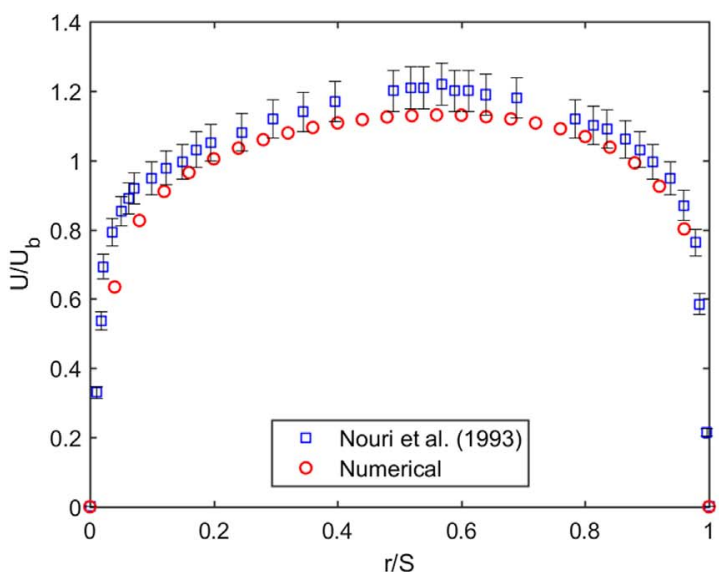

(b)

Fig. 4. Comparison between numerical predictions and experimental data: (a) Custódio (2020) and (b) Nouri et al. (1993).

results from the velocity fields were obtained. Figure 5a shows the axial velocity profile, in a cross section exactly in the region of the intake, in three different positions (lines 1-3). The dashed lines indicate the location of the walls of the inner tube and the outer tube. Since the fluid in the annular region flows through the orifices, which have a smaller area, the axial velocity increases. In addition, a small difference between the profiles can be observed. In the inner tube $(-0.04 \mathrm{~m} \leq r \leq 0.04 \mathrm{~m})$, this small difference is attributed to the shock of the fluid jets that flow through the orifices, and are in the central region (inner region). In the external part (annular region), the difference in the velocity profiles is attributed to the secondary flow that occurs upstream of the intake and which will be presented and discussed further. Both phenomena make the flow asymmetrical in the intake region. Positive axial velocity values, such as those found near the capsule wall, indicate that the part of the fluid in that region does run through the orifices. This analysis is supported by the evaluation of the radial velocity (Fig. 5b), which shows that of velocity close to the capsule wall is approximately zero. Under a gas-liquid flow condition, the combined effect of these two velocity components on a gas bubble in the region close to the capsule wall, would push the bubble into the capsule (downstream of the intake) and not into the intake.

Negative values of axial velocity in the inner region show the fluid recirculation. In this region, upstream of the intake, the internal region behaves as an open cavity, as shown in the current lines in Figure 6.

\subsection{Axial and radial velocity distribution at annular region}

To evaluate the axial distribution of the temporal average of the axial and radial velocities at the six orifices, six lines were created at the axial direction of the main flow for an absolute radial distance of $0.045 \mathrm{~m}$, in annular region, as displayed in Figure 7. The temporal average of the axial and radial velocity distribution is displayed in Figures $7 \mathrm{a}$ and $7 \mathrm{~b}$, respectively.

As shown in Figure 7a, the axial velocity decreases exactly at the orifice's region reaching zero value in $2.04 \mathrm{~m}$ and, after that point, a valley is formed. The axial velocity distribution reaches zero value at the same point for all lines, in approximately $2.35 \mathrm{~m}$, where the fluid is stagnant to the end of the capsule annular. The differences 


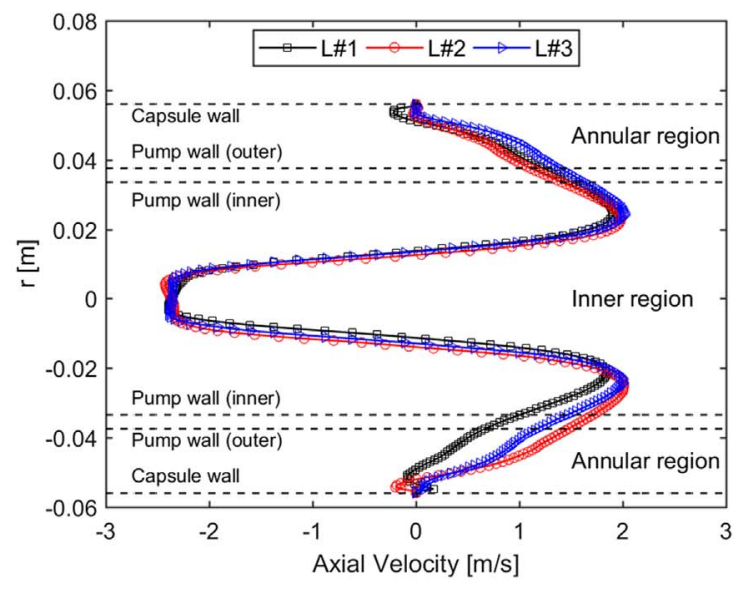

(a)

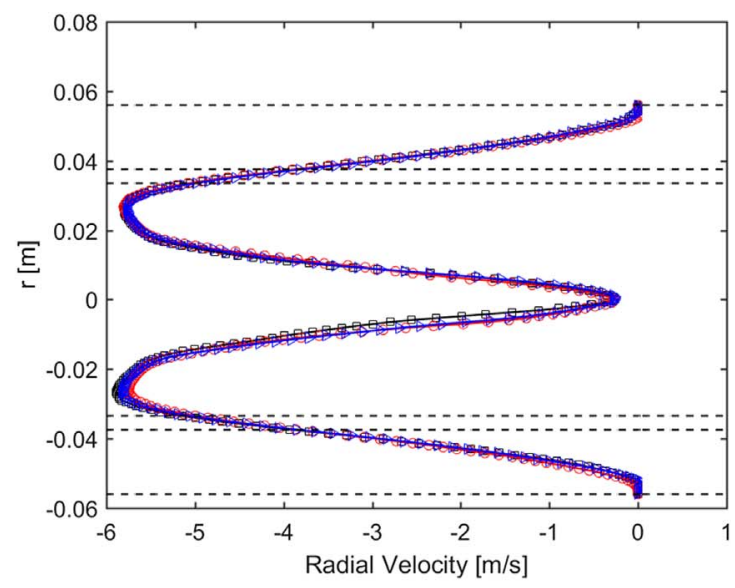

(b)

Fig. 5. Profile of the temporal average component velocities at the orifices: (a) axial velocity and (b) radial velocity. The legends are the same as in Figure 5a.

between the lines upstream to the orifice are lower than downstream, and this occurs due to the secondary flow at the downstream region, with recirculation and vortex structures. Negative axial velocity values indicate that the flow direction is contrary to the " $Z$ " axis, the fluid is returning and flowing into the orifices, this phenomenon occurs for all lines until approximately $2.07 \mathrm{~m}$. The non-symmetry of the flow can also be observed in Figure 7, when comparing the opposite pairs of orifices $(1-4,2-5$ and 3-6).

As it approaches the orifices, the radial velocity increases towards the center of the tube (negative values). The radial velocity reaches the maximum absolute value of $1.5 \mathrm{~m} / \mathrm{s}$ for lines 1 and $4,1.3 \mathrm{~m} / \mathrm{s}$ for lines 5 and 6 and $1.2 \mathrm{~m} / \mathrm{s}$ for lines 2 and 3 . In addition to observing that the radial velocity profiles are asymmetrical at the orifices region, we also note from Figure $6 \mathrm{~b}$ that the liquid flow is not uniform when running through the orifices, since the radial component of the velocity (normal to the orifices) is not uniform. The differences between the values of the radial velocity downstream the orifices are due to the secondary flow at that region.

Comparing Figures $7 \mathrm{a}$ and $7 \mathrm{~b}$, we note that between the dashed lines (intake region), while the axial velocity decreases, the radial velocity increases. Although the radial component of the velocity predominates in this region, the axial velocity cannot be considered null as its value is different from zero.

The results in Figure 7 show that the fluid stagnates approximately $20 \mathrm{~cm}$ from the intake and not immediately upstream of the intake, as is normally found in the literature when modeling the natural separation of fluids (Liu and Prado, 2004; Marquez and Prado, 2003). In addition, the result shows that the flow is asymmetrical in the intake and upstream of the intake region.

\subsection{Intake flow analysis}

Streamlines were obtained to investigate the flow at the intake region, and these are displayed in Figure 8.

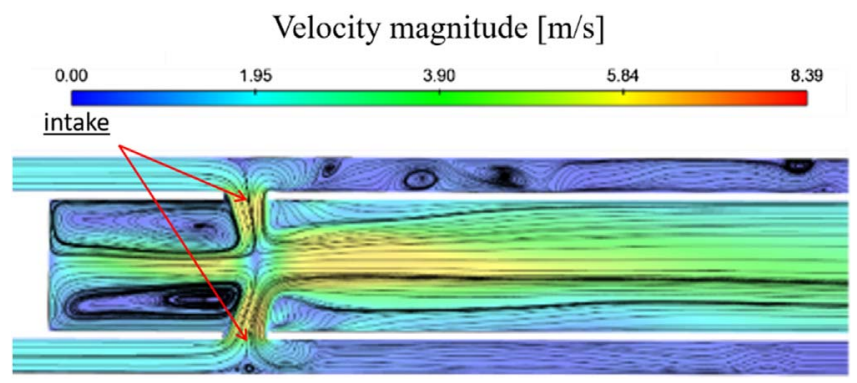

Fig. 6. Inner tube streamlines at intake upstream.

The secondary flow is accentuated in the intake region and, due to the adverse gradient pressure, this region is dominated by vortices. The vortex dynamics can be observed from the comparison of the streamlines over time, with different sizes and quantities at each time-step. At the annular capsule region, eleven vortex structures are observed for time equals to $4 \mathrm{~s}$, while the only 6 structures are observed for $3 \mathrm{~s}$ of flow.

The behavior of the streamlines in the annular region of the intake, displayed in Figure 8, are different from the streamlines when it is assumed that the flow is two-dimensional in a steady state condition. It is evident that upstream from the center of the holes, the secondary flow disturbs the flow in the region of the intake and, consequently, alters the streamlines. A portion of the flow in the capsule upstream of the intake returns and enters the intake towards the pump. From the results presented above, it is expected that the amount of gas that does not pass through the intake towards the pump would be trapped at the top of the capsule, as suggested in the Figure 8.

The vortex generated in the orifices is like the bathtub vortex type and can be better visualized in Figures 9-13. Figure 9 presents the location of the surface and the streamlines and a vortex pair is observed in the orifice 1 for time equals to $5 \mathrm{~s}$. The vortices rotate in opposite directions 


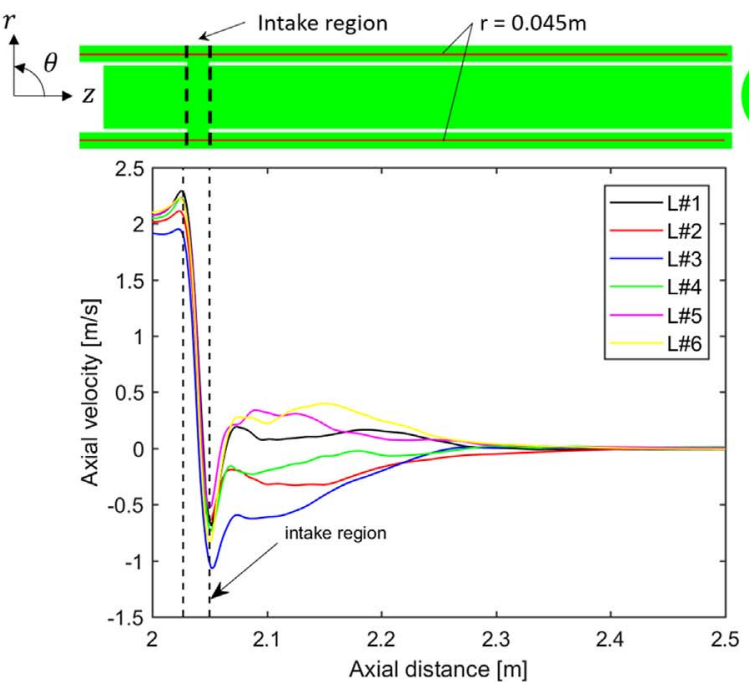

(a)

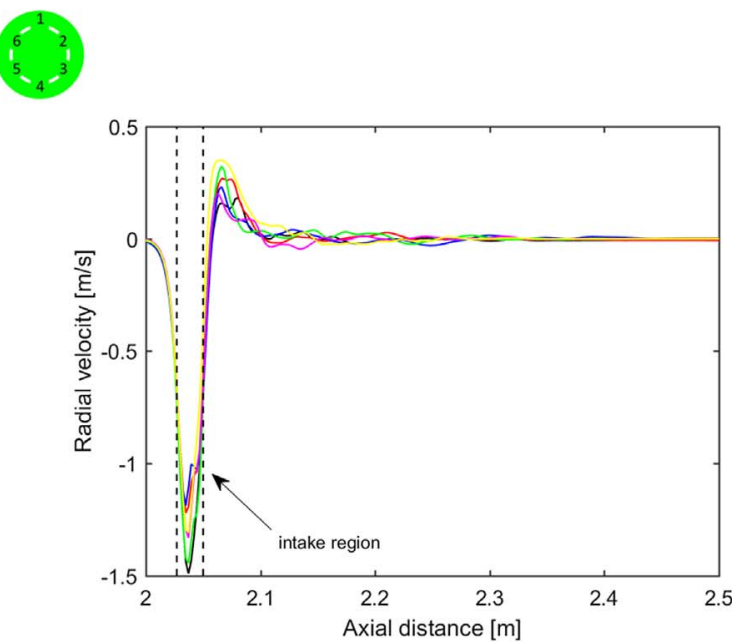

(b)

Fig. 7. Velocity distribution at the six lines: (a) axial velocity and (b) radial velocity. The legends are the same as in Figure $7 \mathrm{a}$.

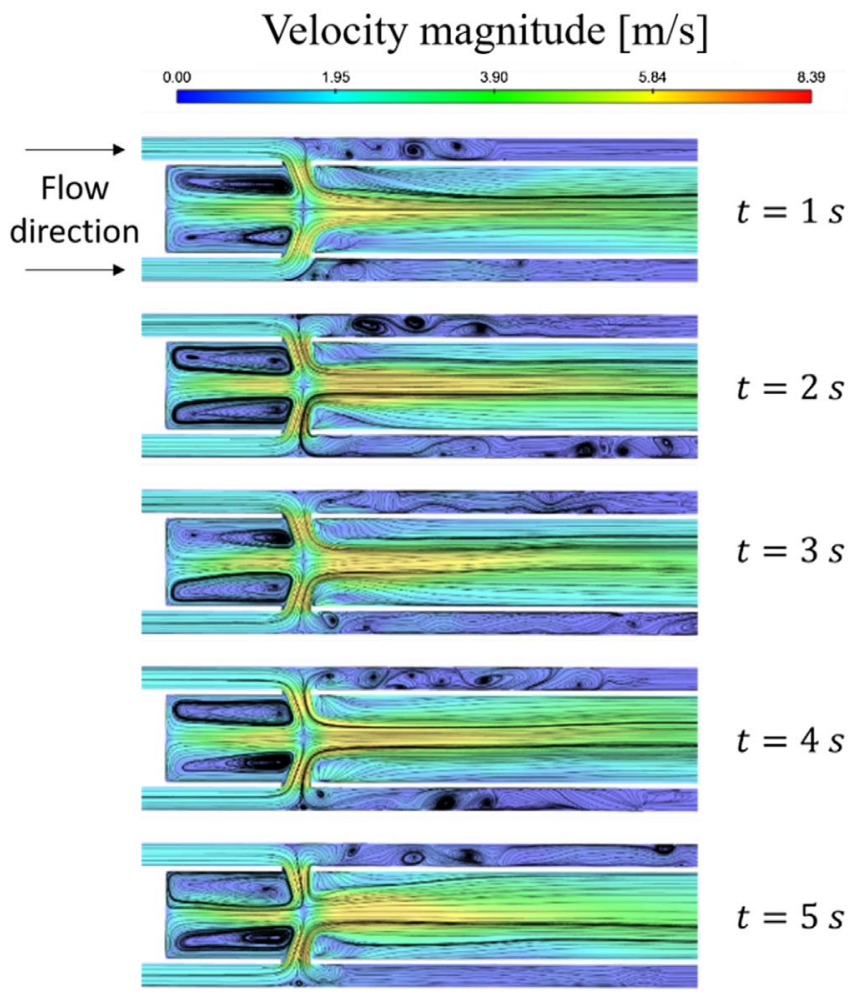

Fig. 8. Streamlines and velocity distribution at the intake region. The plane shows orifices 1 and 4 .

(the rotation direction is represented by the vectors) and the flow direction is positive in the $z$-axis.

Figure 10 presents the vortex motion from 1 to $5 \mathrm{~s}$ in time-steps of $1 \mathrm{~s}$, as well as the vortex pair structure formation. In the first moment, Figure 10 shows that only one vortex structure appears near the orifice wall for $t=1 \mathrm{~s}$. In turn, for $t=2 \mathrm{~s}$, there is a slight movement towards

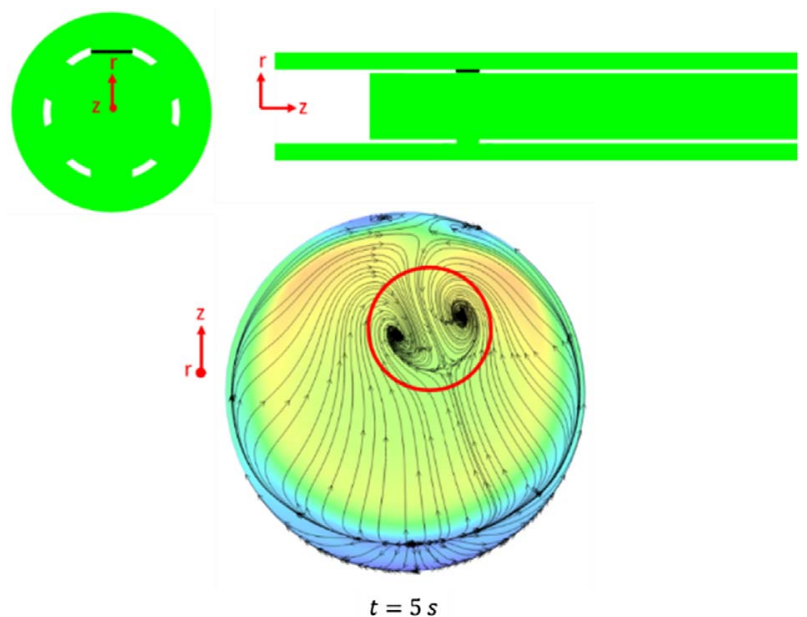

Fig. 9. Vortex pair located in orifice 1 for $5 \mathrm{~s}$ of simulation.

the center of the orifice. At the third time-step, the vortex pair is created and the vectors initially present opposite rotation directions. For $t=5 \mathrm{~s}$, it is possible to note that the vortex pair structure is well defined.

The recirculation of fluid in the capsule upstream of the intake and the presence of vortices in the region of the intake were observed in experimental studies containing gas-liquid two-phase flow (Custódio, 2020; Vieira, 2018), as shown in Figure 11, and the yellow arrows highlight the recirculation and the vortex. Through images, the authors showed vortices of different sizes and in different holes over time, favoring asymmetrical flow in the intake region, as displayed in Figure 12. Numerical results also capture different vortices in different holes, at the same total time, as shown in Figure 13.

The formation of the vortex structure is displayed in Figure 14 in different radial locations. The vortex pair structure is only clearly defined in $r=0.04 \mathrm{~m}$, slightly above 

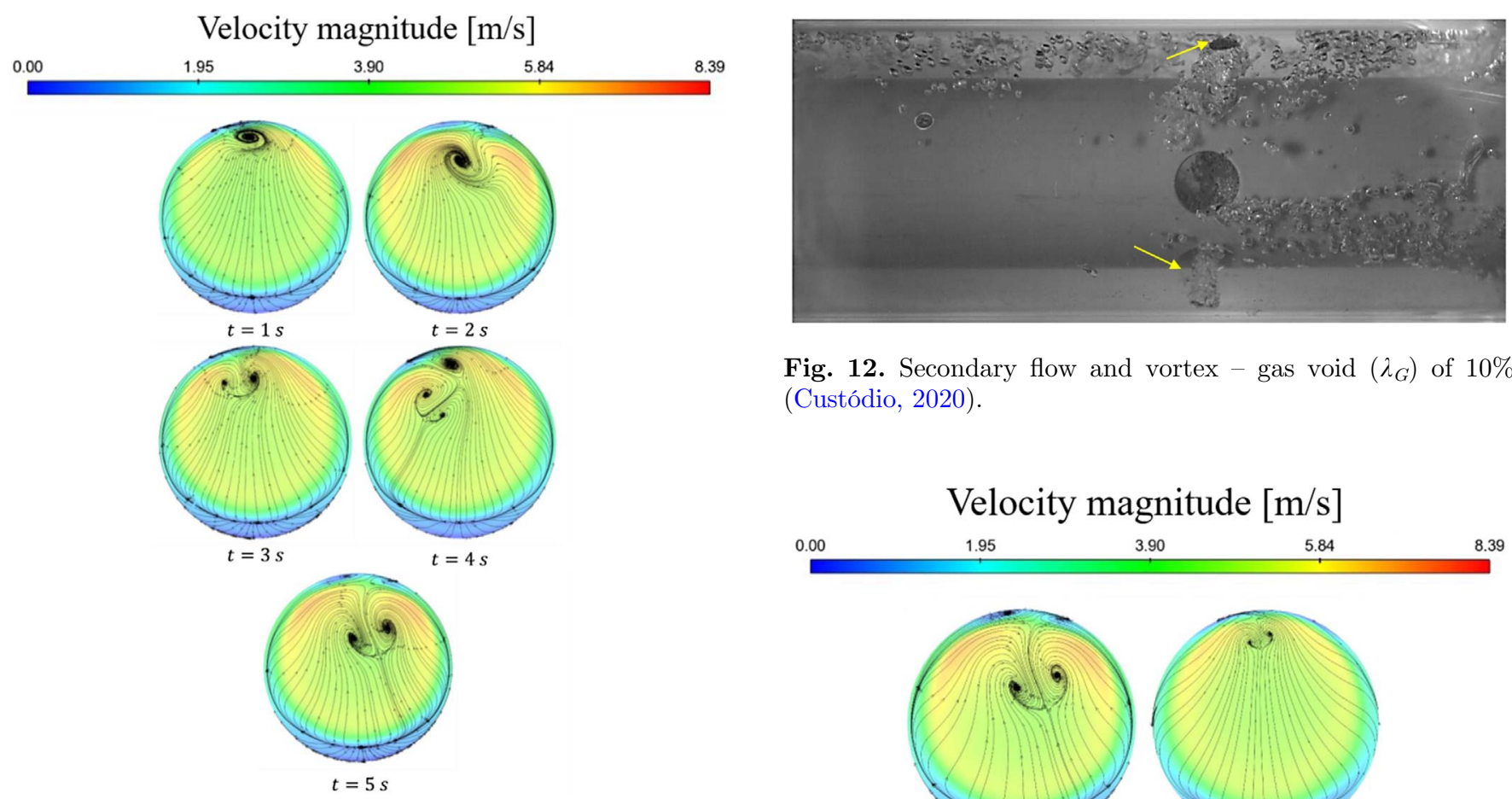

Fig. 12. Secondary flow and vortex - gas void $\left(\lambda_{G}\right)$ of $10 \%$ (Custódio, 2020).

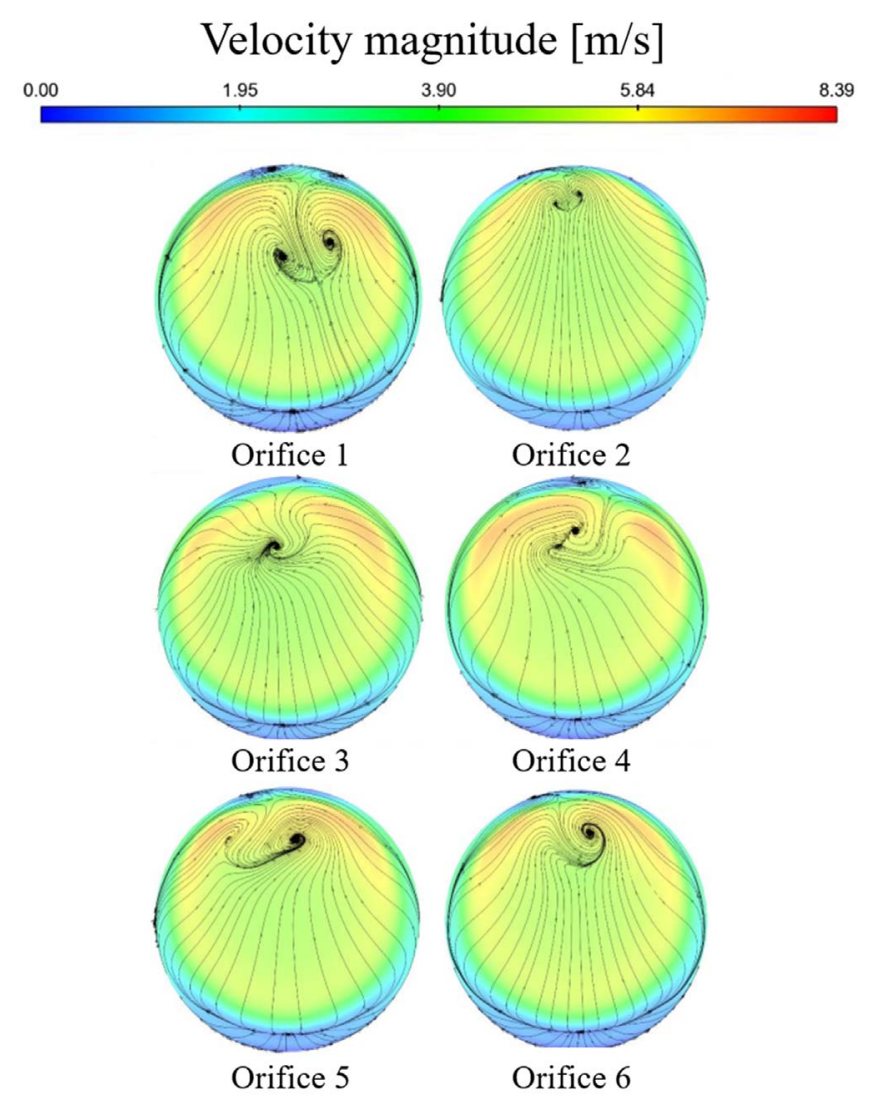

the orifice's inlet. As the vortex pair approaches the center of the tube, it tends to go toward the wall of the orifice. We also note that there is a hydraulic barrier (on top of surfaces $a, b$ and $c$ ) in the flow upstream of the orifices causing the fluid to return to the orifice. At radial distance equals to $0.029 \mathrm{~m}$, the fluid is in the inner tube (flowed through the orifice) and a change in the vortex structure is verified due to the influence of the flow from the other orifices.

The region just above the orifices is a region of low pressure, as displayed in Figure 15, and leads to the creation of a hydraulic barrier, as shown with vorticity contours in Figure 16a. This phenomenon was also verified experimentally by Custódio (2020), as displayed in Figure 16b.

In real application of the ESP-S system, the flow pattern normally found in the region in the intake upstream annular region is the slug flow pattern (Vieira et al., 2020), and system is subject to fluid segregation inside the capsule

Fig. 13. Streamlines, total velocity field and vortex for different orifices for $5 \mathrm{~s}$.

and, mainly, in the intake region. This segregation is known as natural separation and its effects on the performance of submersible centrifugal pumps have been investigated by several researchers (Alhanati, 1993; Harun et al., 2000; Lea and Bearden, 1982; Liu and Prado, 2004; Marquez and Prado, 2003; Vieira et al., 2015, 2020), and models to quantify the separation have been proposed. The most common approach used in segregation models assumes two-dimensional, axisymmetric flow, in a permanent regime. In addition, the models consider that the liquid is totally stagnant in the capsule upstream of the intake. The results presented above show that the flow in the intake region is dominated by vortices and recirculation, 

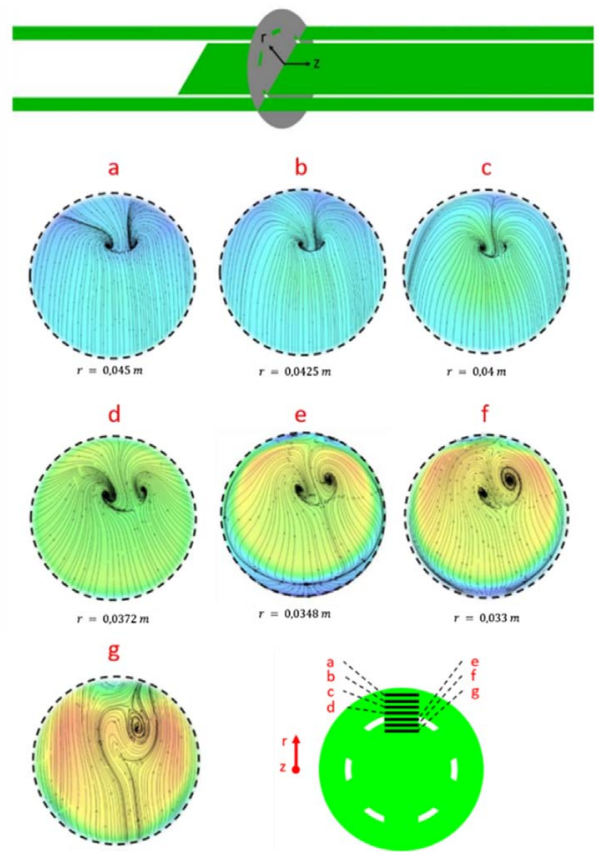

Velocity magnitude $[\mathrm{m} / \mathrm{s}]$

0.00

1.95

3.90

5.84

8.39

Fig. 14. Streamlines for different radial distances for $5 \mathrm{~s}$.

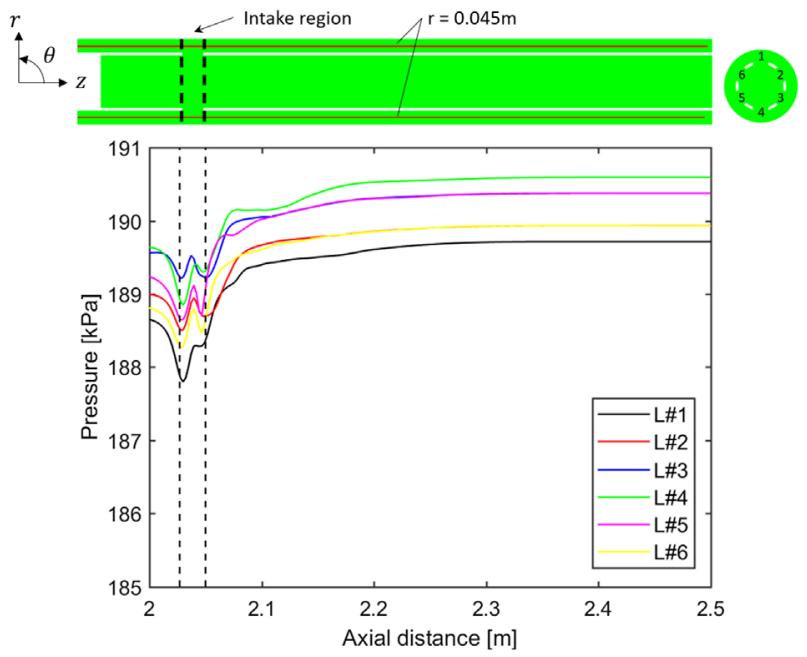

Fig. 15. Pressure drop at the six lines.

which are asymmetrical and unsteady. Experimental studies carried out by Custódio (2020) confirm the existence of vortices and recirculation as well as flow asymmetry.

It is expected that the vortices characterized by the present work and supported by experimental studies, will influence the flow pattern in the intake region, breaking the larger bubbles into smaller bubbles, making the transition from the slug flow pattern to the dispersed bubbles or bubbly pattern. Smaller bubbles would have more difficulty being dragged into the intake and harmful to

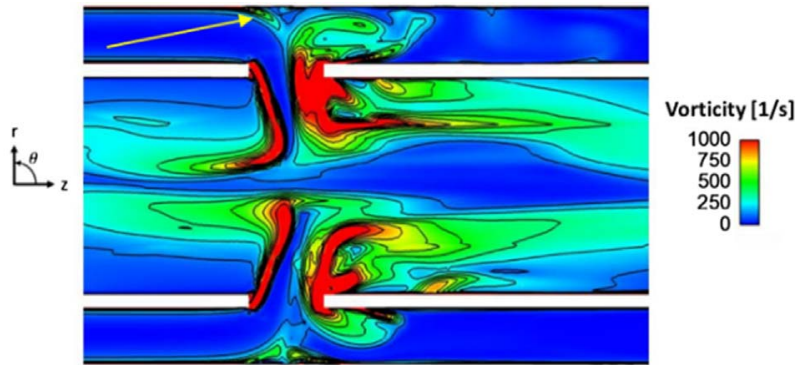

(a)

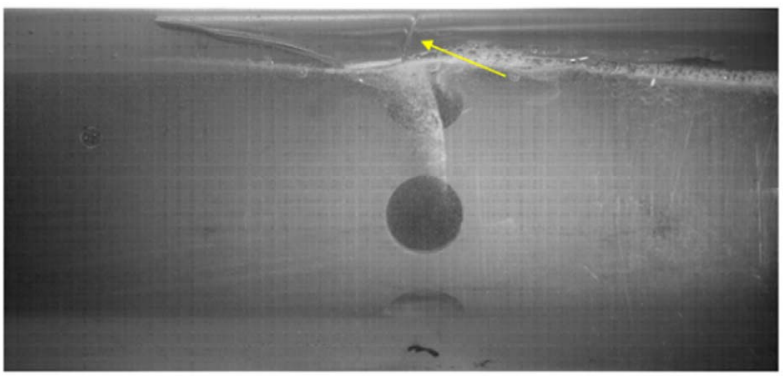

(b)

Fig. 16. Hydraulic barrier: (a) vorticity contours and (b) low pressure region (Custódio 2020).

\section{$Q\left[s^{2}\right]$}

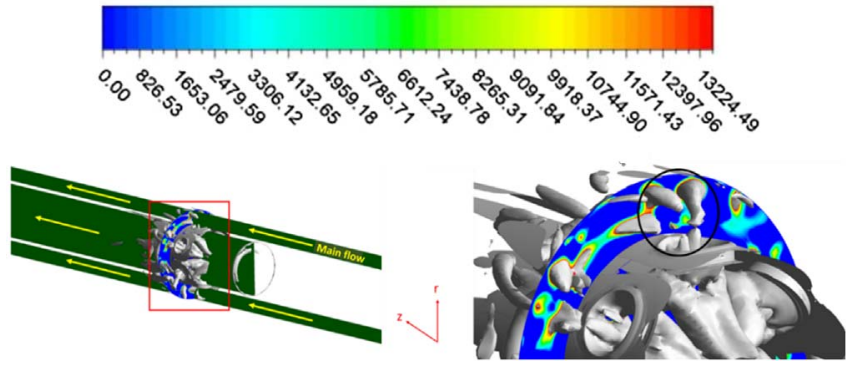

Fig. 17. Three-dimensional vortex structure and $Q$ distribution in a surface for $t=5 \mathrm{~s}$.

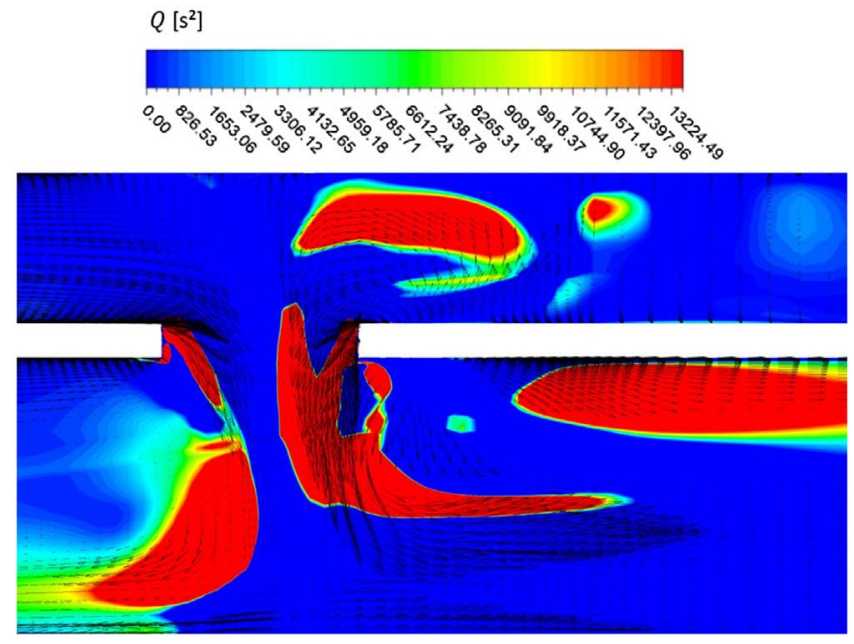

Fig. 18. Vectors and $Q$ distribution in an isometric surface. 


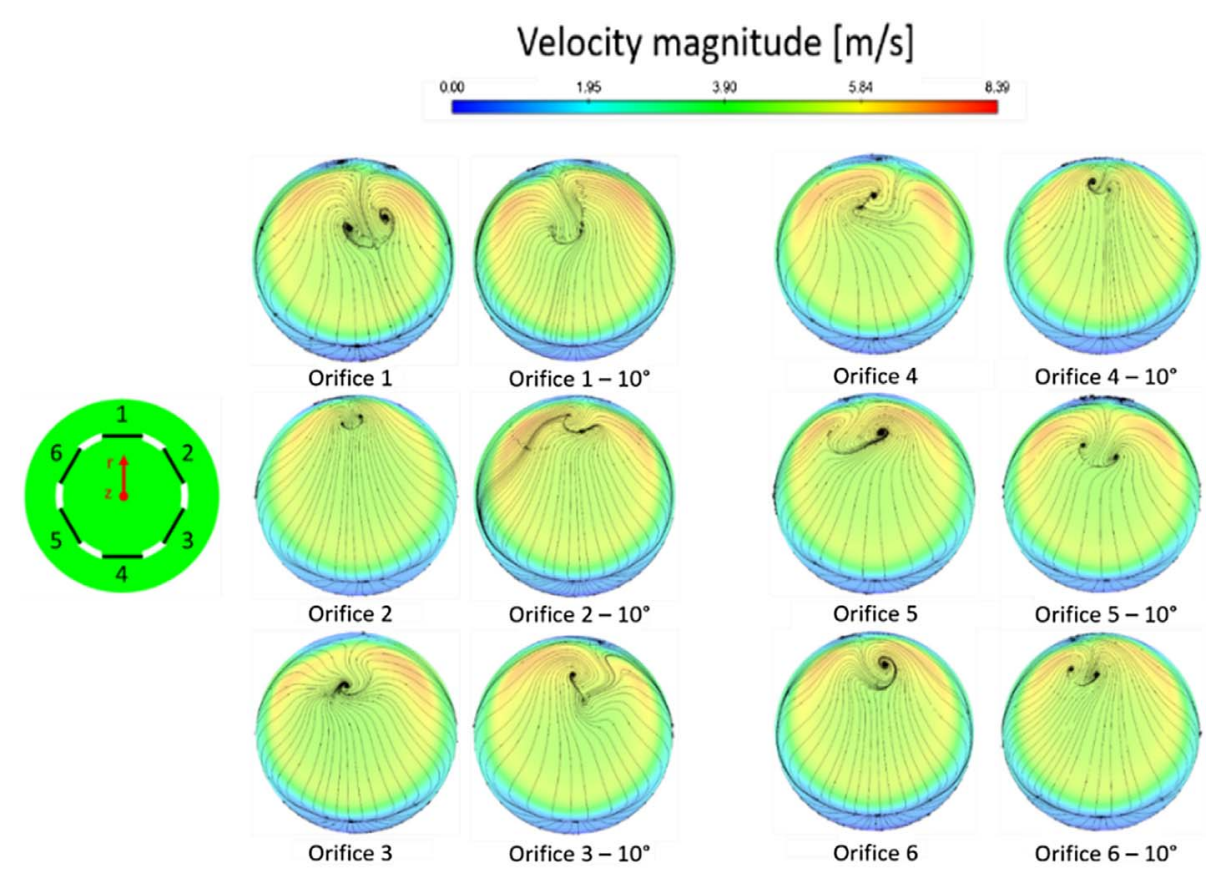

Fig. 19. Streamlines in the intake's orifices for the horizontal and inclined cases.

gas separation. Another harmful consequence of changing the flow pattern in the region of intake is the possibility of gas accumulation at the top of the capsule (after intake). At the limit, the gas boundary can reach the pump intake affecting the pump performance negatively, reducing the liquid rates and the pressure added by the pump (Bagci et al., 2010; Monte Verde, 2011). Consequently, this gas accumulation can lead to the ESP-S shut down operation.

In order to visualize the $3 \mathrm{D}$ vortex structure, we used the method proposed by Hunt et al. (1988), with the $Q$ criterion. The $Q$ criterion was used by Škerlavaj et al. (2014) to predict vortex surfaces in a single-phase simulation. Phillips (2003) defines the $Q$ criterion as an efficient tool to visualize vortex structures. This methodology is used to determine vortex structure in incompressible flows from the magnitude of the vorticity rate, $\Omega$, and the magnitude of the strain rate, $S$, where the vortices are defined in the regions where $\Omega>S$ (Kolár, 2007), so that $Q$ in equation (17) is positive,

$$
Q=\frac{1}{2}\left(\|\Omega\|^{2}-\|S\|^{2}\right) .
$$

A 3D surface is shown in Figure 17 at the orifice's region using the $Q$ criterion and the $Q$ distribution in a surface at $z=2.07 \mathrm{~m}$ from the inlet, for the flow close to the orifice 1. Figure 17 shows that the vortex pair found at the orifice region, shown in Figure 8, is also verified in the annular region. It flows in the contrary direction of the capsule's annular flow and enters the orifice, following towards the outlet. The visualization of the structure after it runs through the orifice follows in Figure 17, where the distribution of $Q$ and vectors are presented in an isometric surface close to orifice 1 . Contours of $Q$ values show that the magnitude of the vorticity is greater than the magnitude of the strain rate and indicate the presence of vortex.
When running through orifice 1 , this vortex pair structure maintains its direction in a main flow-favorable direction, forming a " $C$ " shape as shown in Figure 18.

Figure 18 is a complement to Figure 17 and shows, in a $2 \mathrm{D}$ plane, the magnitude of $Q$ and the vortices' location. Figure 18 allows you to view the vortices in the region of intake and inside the inner tube. This view is quite restricted in Figure 17. The red area indicates where the magnitude of the vorticity (Fig. 16) is much higher than the magnitude of the strain rate. The vortices are located near the edge of the holes, in the direction of flow.

\subsection{Effects of inclination on vortex structures}

Considering the irregularities of the marine ocean floor, the ESP-S must be installed slightly inclined $\left(5^{\circ} \sim 10^{\circ}\right)$. The effects of inclination on gas-liquid flow was investigated by Custódio (2020), who observed a transition of fluid patterns with the inclination angle increase.

The influence of the inclination on the structure of the vortices in each intake's orifice is displayed in Figure 19, for $t=5 \mathrm{~s}$, where the horizontal flow condition is compared with inclined flow condition for $\alpha=10^{\circ}$. The pressure distribution is influenced by the change in the gravitational field, due to the system's inclination. Because of this, it can be seen from Figure 19, that the inclination affects the structure of the vortices in all six orifices when compared to a horizontal flow condition.

\section{Conclusion}

In this work, a numerical study was carried out by using a Finite Volume Method to characterize the capsule-intake 
single-phase turbulent flow of the scaled ESP-S. The numerical model was validated with experimental data, axial velocity and pressure drop, from the literature and a qualitative comparison of vortices and recirculation in the intake region was performed with two-phase gas-liquid flow. The following conclusions can be drawn after the detailed analysis:

1. The numerical results demonstrated that the intake region plays an important role in the flow, as it has instabilities such as recirculation zones and vortex structures. The results demonstrated that the velocity field is completely changed by this region precisely due to these instabilities.

2. The vortices in the intake region are dynamic, both in relation to the same orifice and in different orifices. Under the time of analysis considered, structures of up to three vortexes were observed, as shown in Figures 10 and 13.

3. The vortex generated in the orifices is like the bathtub vortex type and just above the orifices is a region of low pressure leading to the creation of a hydraulic barrier, as shown with vorticity contours in Figure 16a and experimentally in Figure 16b.

4. The vortex core and its structure are influenced by the inclination angle of the ESP, as shown in Figure 19. It is expected that a change in vortex' structure can lead to a change in the flow pattern in the intake region.

5. Through the velocity fields and the streamlines, we verified that the intake region is dominated by the secondary flow, making the flow asymmetric in the intake region. In addition, the results revealed that the flow is unsteady and that the fluid stagnation point does not occur immediately upstream of the intake, but approximately $20 \mathrm{~cm}$ from the intake. This conclusion is relevant because many natural segregation models consider the flow to be two-dimensional, symmetrical, and steady.

6 . The present single-phase study suggests that conclusions 1-5 will impact two-phase gas-liquid flow, mainly in the region of the pump intake. It is expected that the vortices characterized by the present work and supported by experimental studies, will influence the flow pattern in the intake region, breaking the larger bubbles into smaller bubbles, making the transition from the slug flow pattern to the dispersed bubbles or bubbly pattern. Smaller bubbles would have more difficulty being dragged into the intake and harmful to natural gas segregation. Since a certain amount of gas does not enter the intake, it will accumulate at the top of the capsule and, at the limit, the gas boundary can reach the pump intake affecting the pump performance negatively, reducing the liquid rates and the pressure added by the pump. Consequently, this gas accumulation can lead to the ESP-S shut down operation.

This study is fundamental to understanding the complex physical phenomena involved in the capsule-intake flow within the ESP-S. Although the investigation performed is single-phase with water and disregards the motor, the shaft and the pump, quite different from the real application, the numerical model is considered to capture the main characteristics of the flow in the capsule-intake region. It is recommended to evaluate the influence of shaft in future work, together with the presence of the pump. The conclusions found in this work will be used to extend the study for the two-phase gas-liquid flow, aiming to generate a tool to assist in the equipment's dimensioning and optimization.

Acknowledgments. The authors wish to thank Petrobras for their financial and support in this study. Also, we would like to thank FAPESP for the scientific support 2017/06978-3.

\section{References}

Alhanati F.J.S. (1993) Bottomhole gas separation efficiency in electrical submersible pump installations, The University of Tulsa, Tulsa, OK.

Azouz I., Shirazi S.A. (1998) Evaluation of several turbulence models for turbulent flow in concentric and eccentric annuli, J. Energy Resour. Technol. 120, 268. https://doi.org/ 10.1115/1.2795047.

Bagci S., Hughes B., Nava J.D. (2010) Challenges of using electrical submersible pump (ESP) in high free gas applications, in: International Oil and Gas Conference and Exhibition in China, Beijing, China. https://doi.org/10.2118/131760MS.

Chung S.Y., Rhee G.H., Sung H.J. (2002) Direct numerical simulation of turbulent concentric annular pipe flow: Part 1: Flow field, Int. J. Heat Fluid Flow 23, 426-440. https://doi. org/10.1016/S0142-727X(02)00140-6.

Chung S.Y., Sung H.J. (2005) Large-eddy simulation of turbulent flow in a concentric annulus with rotation of an inner cylinder, Int. J. Heat Fluid Flow 26, 191-203. https://doi.org/ 10.1016/J.IJHEATFLUIDFLOW.2004.08.006.

Costa B.M.P., Oliveira P. da S., Roberto M.A.R. (2013) Mudline ESP: Eletrical submersible pump installed in a subsea skid, in: Offshore Technology Conference, Houston, Texas, USA, May 2013. https://doi.org/10.4043/24201-MS.

Custódio D.A.S. (2020) Experimental study of the influence of pipe slope and liquid viscosity on two-phase gas-liquid flow in an annular duct, University of Campinas, Campinas, Brazil.

Harun A.F., Prado M.G., Serrano J.C., Doty D.R. (2000) A simple model to predict natural gas separation efficiency in pumped wells, in: SPE Annual Technical Conference and Exhibition, Society of Petroleum Engineers. https://doi.org/ 10.2118/63045-MS.

Hunt J.R.C., Wray A.A., Moin P. (1988) Eddies, stream, convergence zones in turbulence flows, in: Proceedings of the Summer Program. NTRS - NASA Technical Reports Server.

Kolář V. (2007) Vortex identification: New requirements and limitations, Int. J. Heat Fluid Flow 28, 638-652. https://doi. org/10.1016/j.ijheatfluidflow.2007.03.004.

Lea J.F., Bearden J.L. (1982) Effect of gaseous fluids on submersible pump performance, J. Pet. Technol. (JPT) 34, 2922-2930. https://doi.org/10.2118/9218-PA. 
Liu B., Prado M. (2004) Modeling downhole natural separation using a bubble tracking method, in: American Society of Mechanical Engineers, Pressure Vessels and Piping Division (Publication) PVP, American Society of Mechanical Engineers Digital Collection, pp. 3-10. https://doi.org/10.1115/PVP20042844

Marquez R.A., Prado M.G. (2003) A new robust model for natural separation efficiency, in: SPE Production and Operations Symposium, Society of Petroleum Engineers. https://doi.org/10.2118/80922-MS.

Menter F.R. (1994) Two-equation eddy-viscosity turbulence models for engineering applications, AIAA J. 32, 1598-1605. https://doi.org/10.2514/3.12149.

Monte Verde W. (2011) Experimental investigation of two-phase gas-liquid flow performance of electrical submersible pump, University of Campinas, Campinas, Brazil.

Monte Verde W., Biazussi J.L., Sassim N.A., Bannwart A.C. (2017) Experimental study of gas-liquid two-phase flow patterns within centrifugal pumps impellers, Exp. Therm. Fluid Sci. 85, 37-51. https://doi.org/10.1016/j.expthermflusci.2017.02.019.

Neto J.L.V., Martins A.L., Neto A.S., Ataíde C.H., Barrozo M. A.S. (2011) CFD applied to turbulent flows in concentric and eccentric annuli with inner shaft rotation, Can. J. Chem. Eng. 89, 636-646. https://doi.org/10.1002/cjce.20522.

Nouri J.M., Umur H., Whitelaw J.H. (1993) Flow of newtonian and non-newtonian fluids in concentric and eccentric annuli, J. Fluid Mech. 253, 617. https://doi.org/10.1017/ S0022112093001922.

Okumura T., Merzari E., Ninokata H. (2006) Direct numerical simulation of turbulent flows in an eccentric annulus channel, Trans. Am. Nucl. Soc. 95, 825-826.

Patankar S.V. (1980) Numerical heat transfer and fluid flow, Hemisphere Publishing Corporation, New York.

Patankar S.V. (1981) A calculation procedure for two-dimensional elliptic situations, Numer. Heat Transf. 4, 409-425. https://doi.org/10.1080/01495728108961801.

Patankar S.V., Spalding D.B. (1972) A calculation procedure for heat, mass and momentum transfer in three-dimensional parabolic flows, Int. J. Heat Mass Transf. 15, 1787-1806. https://doi.org/10.1016/0017-9310(72)90054-3.

Perissinotto R.M., Monte Verde W., de Castro M.S., Biazussi J. L., Estevam V., Bannwart A.C. (2019a) Experimental investigation of oil drops behavior in dispersed oil-water two-phase flow within a centrifugal pump impeller, Exp. Therm. Fluid Sci. 105, 11-26. https://doi.org/10.1016/j.expthermflusci. 2019.03.009.

Perissinotto R.M., Monte Verde W., Gallassi M., Gonçalves G. F.N., de Castro M.S., Carneiro J., Biazussi J.L., Bannwart A. C. (2019b) Experimental and numerical study of oil drop motion within an ESP impeller, J. Pet. Sci. Eng. 175, 881895. https://doi.org/10.1016/j.petrol.2019.01.025.

Phillips O.M. (2003) Shear-flow turbulence, in: Annual Review of Fluid Mechanics. Fluid Mechanics and Its Applications, Johns Hopkins University, Baltimore, Maryland, pp. 245264. https://doi.org/10.1146/annurev.fl.01.010169.001333.

Pineda H., Biazussi J., López F., Oliveira B., Carvalho R.D.M., Bannwart A.C., Ratkovich N. (2016) Phase distribution analysis in an Electrical Submersible Pump (ESP) inlet handling water-air two-phase flow using Computational Fluid Dynamics (CFD), J. Pet. Sci. Eng. 139, 49-61. https://doi. org $/ 10.1016 /$ j.petrol.2015.12.013.
Roache P.J. (1994) Perspective: A method for uniform reporting of grid refinement studies, J. Fluids Eng. 116, 405. https://doi.org/10.1115/1.2910291.

Shi Y., Zhu H., Zhang Jinya, Zhang Jiate, Zhao J. (2018) Experiment and numerical study of a new generation threestage multiphase pump, J. Pet. Sci. Eng. 169, 471-484. https://doi.org/10.1016/j.petrol.2018.06.011.

Silva L.C.T. (2010) Numeric Simulation of a Dummy Well Pumping Module, School of Chemistry, Federal University of Rio de Janeiro, Rio de Janeiro.

Škerlavaj A., Škerget L., Ravnik J., Lipej A. (2014) Predicting free-surface vortices with single-phase simulations, Eng. Appl. Comput. Fluid Mech. 8, 193-210. https://doi.org/10.1080/ 19942060.2014.11015507.

Tarcha B.A., Borges O.C., Furtado R.G. (2015) ESP installed in a iubsea skid at Jubarte field, in: SPE Artificial Lift Conference - Latin America and Caribbean, Society of Petroleum Engineers. https://doi.org/10.2118/173931-MS.

Torii S., Yang W.-J. (1994) Numerical study on turbulent flow and heat transfer in circular couette flows, Numer. Heat Transf. Part A Appl. 26, 321-336. https://doi.org/10.1080/ 10407789408955995.

Van Zyl W.R., Dirker J., Meyer J.P. (2013) Single-phase convective heat transfer and pressure drop coefficients in concentric annuli, Heat Transf. Eng. 34, 1112-1123. https://doi.org/10.1080/01457632.2013.763550.

Vieira S.C. (2018) Separação Gás-Líquido no Escoamento Bifásico Intermitente em um Duto Anular, University of Campinas, Campinas, Brazil.

Vieira T.S., Siqueira J.R., Bueno A.D., Morales R.E.M., Estevam V. (2015) Analytical study of pressure losses and fluid viscosity effects on pump performance during monophase flow inside an ESP stage, J. Pet. Sci. Eng. 127, 245-258. https://doi.org/10.1016/j.petrol.2015.01.014.

Vieira S.C., van der Geest C., Fabro A.T., de Castro M.S., Bannwart A.C. (2020) Experimental study and modelling of developing intermittent slug flow in inclined annular pipelines, J. Pet. Sci. Eng. 192, 107204. https://doi.org/10.1016/j. petrol.2020.107204.

Zhang W., Yu Z., Li Y. (2018) Analysis of flow and phase interaction characteristics in a gas-liquid two-phase pump, Oil Gas Sci. Technol. - Rev. IFP Energies nouvelles 73, 69. https://doi.org/10.2516/ogst/2018072.

Zhang W., Yu Z., Li Y., Yang J., Ye Q. (2019) Numerical analysis of pressure fluctuation in a multiphase rotodynamic pump with air-water two-phase flow, Oil Gas Sci. Technol. - Rev. IFP Energies nouvelles 74, 18. https://doi.org/10.2516/ogst/ 2018101.

Zhu J., Banjar H., Xia Z., Zhang H.Q. (2016) CFD simulation and experimental study of oil viscosity effect on multi-stage Electrical Submersible Pump (ESP) performance, J. Pet. Sci. Eng. 146, 735-745. https://doi.org/10.1016/j.petrol.2016.07.033.

Zhu J., Zhu H., Cao G., Zhang J., Peng J., Banjar H., Zhang H.Q. (2019) A New mechanistic model to predict boosting pressure of electrical submersible pumps ESPs under highviscosity fluid flow with validations by experimental data, in: SPE Gulf Coast Section Electric Submersible Pumps Symposium, Society of Petroleum Engineers. https://doi.org/ 10.2118/194384-MS. 